\title{
G
}

Luis Manuel Ruiz Gómez*

Laura Rodríguez Fernández **

César Muñoz Martínez***

\section{APLICACIÓN DE TECNOLOGÍAS DE LA COMUNICACIÓN EN EL SECTOR TURÍSTICO ESPAÑOL}

Internet y los avances en las tecnologías de la comunicación están revolucionado la industria del turismo. Se crean nuevas herramientas para conocer los gustos de los consumidores y saber su opinión; también aparecen nuevos canales de comunicación entre los agentes del sector. Este artículo va analizar estos cambios, cómo estos nuevos instrumentos hacen cambiar la manera de trabajar de las empresas y cómo los clientes hacen uso de las nuevas tecnologías en el mercado turístico español. Como resultado mostraremos cómo se están incorporando estos cambios en la manera de trabajar en las empresas y en la manera de demandar de los consumidores, junto con las perspectivas de futuro en el sector turístico español, haciendo especial referencia al sector de las pymes.

Palabras clave: turismo, TIC, competitividad.

Clasificación JEL: L83, O3.

\section{Introducción}

Los avances en las tecnologías de la información y comunicación (TIC) han generado importantes modificaciones en el patrón de comportamiento de los agentes económicos y en su interactuación con el entorno social y económico. El desarrollo exponencial de los programas y aplicaciones telemáticas ha generado un nuevo contexto en el que se han desarrollo actividades, canales de información y productos de nueva generación.

En paralelo, estos avances tecnológicos han generado progresos en todos los sectores

* Departamento de Organización de Empresas, UNED.

** Departamento de Economía de la Empresa y Contabilidad, UNED.

*** Departamento de Economía Aplicada, UNED.

Versión de junio de 2016. económicos, que han repercutido de forma positiva en la productividad y organización de las empresas hoteleras, compañías aéreas o agencias de viajes, facilitando la interacción directa con el consumidor a través de Internet.

El proceso de globalización ha configurado un entorno dinámico en el que se torna necesario la búsqueda continua de la innovación de nuevos métodos y técnicas que mejoren las estrategias empresariales. En este nuevo escenario, el uso de las TIC se ha convertido en imperativo para mantener la competitividad de las empresas turísticas, afectando a toda la cadena de valor de las organizaciones, desde la política de recursos humanos a los servicios postventa, pasando por la gestión y las acciones de marketing. 
Considerando la contribución de las TIC al crecimiento del sector, resulta imprescindible analizar el impacto de las mismas en los negocios turísticos, especialmente en las actividades de comercialización y distribución de los servicios. Con este objeto, presentamos este artículo cuyo contenido se estructura en tres partes: en primer lugar, dimensionaremos el sector turístico en nuestro país y presentamos la importancia de las TIC en la industria turística analizando la oferta y la demanda de productos turísticos para, posteriormente, pivotar nuestro análisis sobre la incidencia de las TIC en las pequeñas y medianas empresas; finalmente emitimos una serie de conclusiones.

\section{El impacto de las TIC en el mercado turístico}

Las TIC ayudan también a optimizar el valor del dinero y el tiempo de los consumidores turísticos, facilitando el acceso a un amplio volumen de información sobre productos y destinos turísticos. En este sentido, se puede afirmar que la introducción paulatina de las TIC en las empresas turísticas se debe a las demandas de los clientes. Según Camisón y Muñoz (2015), la importancia y necesidad del uso de las TIC en la industria turística atiende a diferentes razones: la necesidad de obtener una información actualizada, fidedigna y detallada para la promoción y comercialización de productos y servicios, que son bienes intangibles; las oportunidades de extender los negocios turísticos en un ámbito global, facilitando que estas empresas puedan asociarse con otras de todo el mundo de manera rentable y eficiente; la obtención de una mayor eficiencia en la gestión de sus operaciones; la creación de nuevos productos específicos dirigidos a determinados nichos de mercado, permitiendo que estas empresas puedan alcanzar una ventaja competitiva sostenible a través de la diferenciación; la mayor agilidad en la gestión de los procesos que configuran el proceso de servicio turístico; la comunicación directa con el cliente (Customer Relationship Management o CRM), permitiendo a las empresas actuar recíprocamente con sus clientes, introduciendo modificaciones continuas para satisfacer al máximo sus necesidades específicas; la prestación del producto servicio turístico en mejores condiciones, así como la incorporación de nuevos servicios, lo que incrementa la satisfacción del cliente; la mejora de la coordinación y el control de las actividades; el apoyo al proceso de toma de decisiones estratégicas; la reducción de los costes operativos y de operación, así como los costes fijos de personal administrativo y de telecomunicaciones; la aplicación de precios flexibles y competitivos; la anticipación de excesos de capacidad o de demanda, incrementando su rapidez en la acción y respuesta a la misma; y la disminución de los gastos derivados de comisiones a intermediarios, pudiendo controlar el proceso de venta directa desde la creación de una página web propia o pagando cuotas de distribución menores a intermediarios electrónicos.

En consecuencia, el potencial de las TIC no se encuentra limitado a la obtención por parte de la empresa de información sobre el mercado turístico, sino que es una pieza clave a la hora de crear y mantener relaciones recíprocas con sus consumidores y proveedores. Adicionalmente, estas relaciones se realizan de una forma más rápida, flexible y eficiente permitiendo mejorar la gestión de sus operaciones y segmentar en mayor medida sus nichos de mercado. Contrariamente, este contexto genera un entorno más inestable y cambiante por lo que las empresas deben aprender a gestionar el empleo de sus TIC para obtener ventajas competitivas en un sector en constante cambio.

En consecuencia, la aplicación de las nuevas tecnologías de la información y la comunicación en el turismo, ha provocado cambios estructurales profundos en este mercado. En los siguientes puntos, vamos a analizar los efectos generados en el proceso de negocio $D$ 


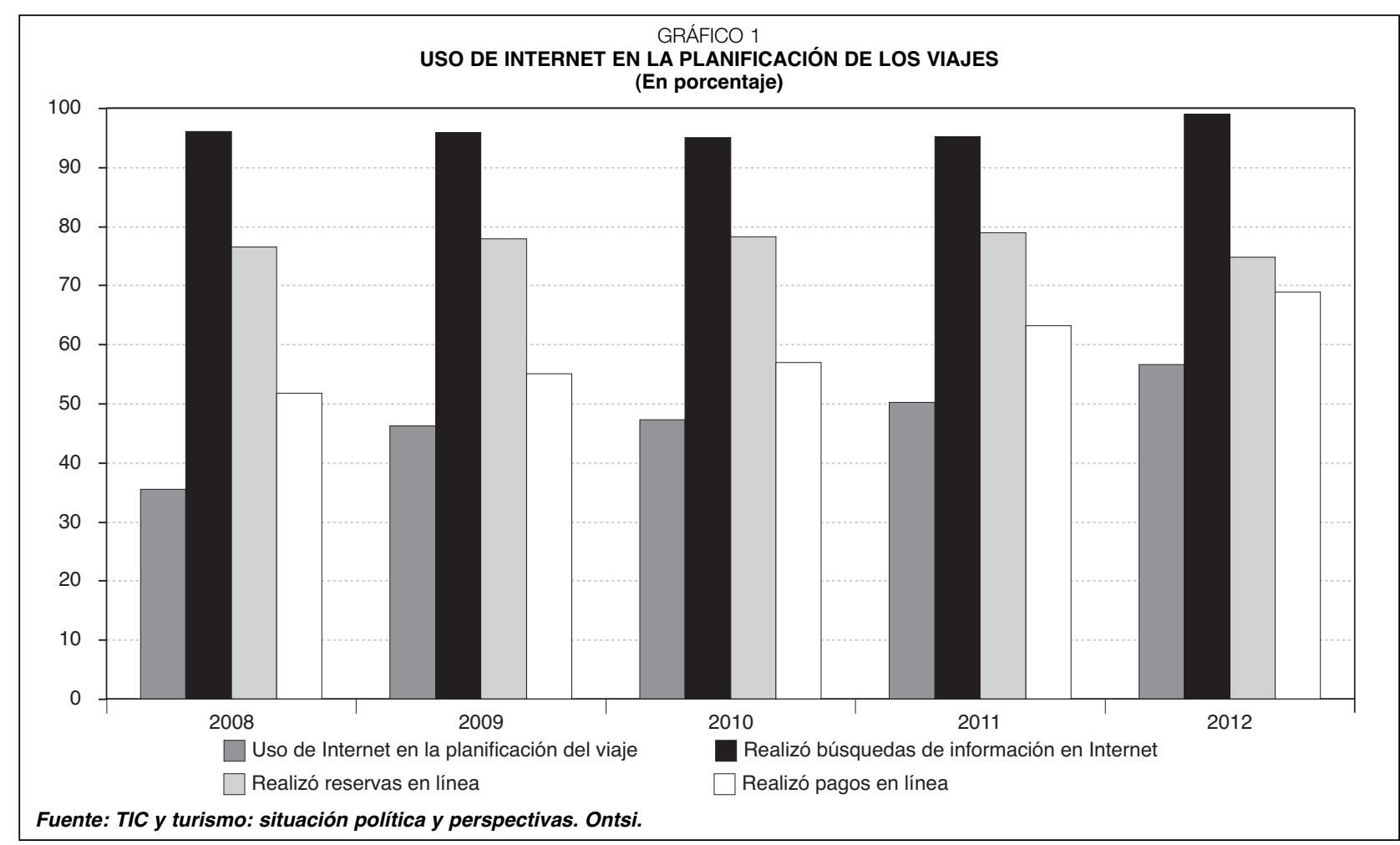

turístico, concretamente en la demanda y oferta de productos turísticos.

\subsection{Demanda turística}

En el caso de la demanda turística la aparición de las TIC ha experimentado una gran transformación, y cada día son más las peticiones de crecimiento de la presencia de estas tecnologías en productos turísticos, ya sea por nuevas aplicaciones, o programas informáticos. El perfil del turista ha cambiado en los últimos años, precisamente por la influencia de las TIC en los procesos de búsqueda de información de los productos; en la actualidad, nos encontramos a un proconsumidor que demanda productos más personalizados y de mayor calidad con el objetivo de maximizar su restricción de ocio-consumo.

A tal efecto, las TIC juegan un papel importante en la transformación de la demanda turística, permitiendo a los consumidores identificar el producto más adecuado a sus necesidades, encontrar valoraciones de las experiencias de otros clientes y dotar de una mayor interactividad en su relación con la oferta turística. Tal y como observamos en el Gráfico 1, la proporción de consumidores que emplean las herramientas online para planificar, reservar o pagar los servicios turísticos registra una proporción muy alta.

Las características especiales de los productos turísticos, intangibilidad y heterogeneidad, impiden conocer la calidad del mismo antes de realizar la compra ni crear un valor de marca pues la calidad del producto depende de múltiples variables relacionadas con el gusto de cada uno de los clientes. En este sentido, la búsqueda y transmisión de la información por parte de los motores de búsqueda de Internet es la parte prioritaria en el mercado; por lo que la demanda de información debe adecuarse de una forma inmediata a las necesidades del consumidor.

\subsection{Oferta}

En el lado de la oferta, Internet ha permitido la conexión de millones de dispositivos a través $\triangleright$ 


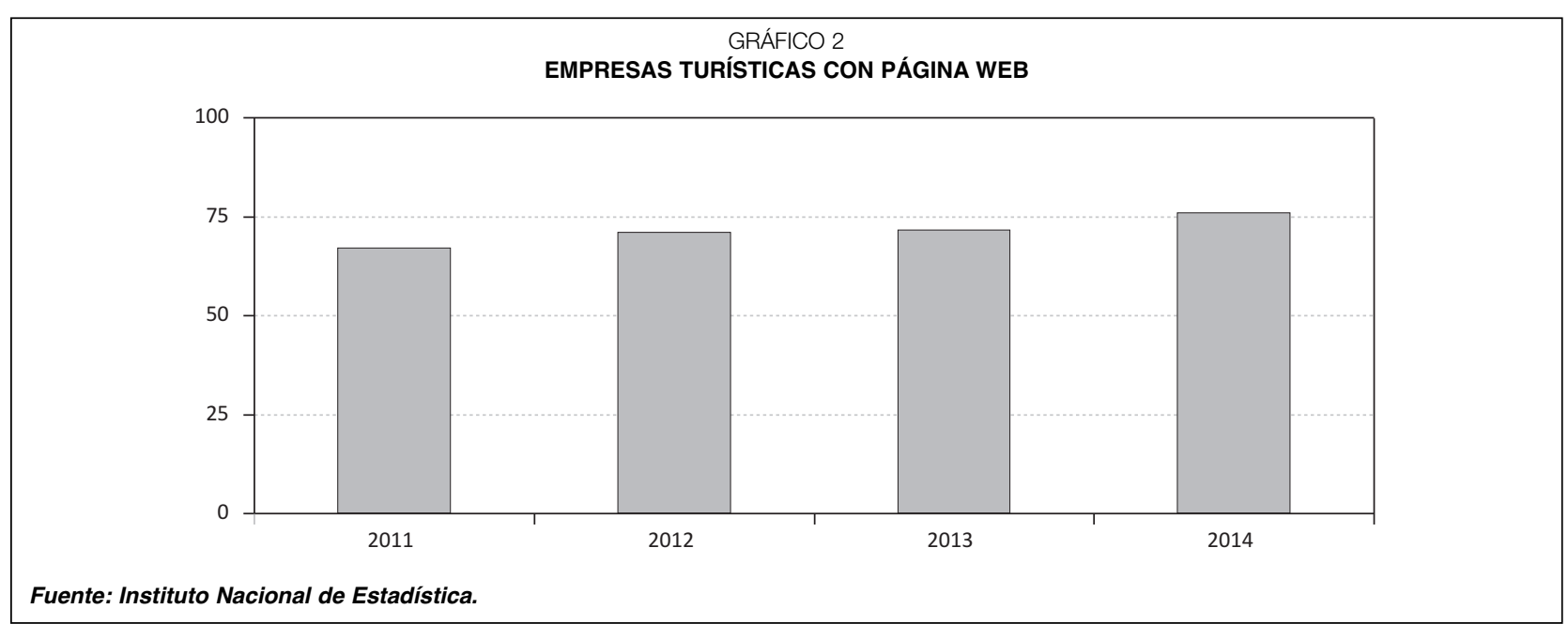

de los cuales los consumidores han tenido acceso de manera instantánea a toda la información que han necesitado sobre el destino, reserva, características del producto y otra serie de características. Todo esto es el resultado del desarrollo de los proveedores turísticos que tratan de satisfacer estas nuevas demandas de los clientes.

Los medios para comunicarse entre el demandante y el oferente de manera directa y eficaz han ido mejorando gracias a las tecnologías de la información. Muchas de estas herramientas están permitiendo a los proveedores de servicios turísticos conocer a sus clientes y permite mejorar mecanismos de distribución y modificaciones en el producto tratándolo de adecuar a las nuevas demandas de los consumidores.

Muchos de estos proveedores son capaces de incorporar de manera automática las ventajas de las tecnologías de la información y de la comunicación siendo capaces de ir ajustando su oferta en función de nuestros patrones de comportamiento en el uso de diversos programas o apps. Esto permite rediseñar ofertas haciéndolas cada vez más eficientes y eficaces.

En la Tabla 1 se muestra cómo los oferentes pueden observar de una manera rápida el comportamiento de los compradores de servicio.

Todos los que conforman parte de la oferta turística (intermediarios, operadores turísticos, alojamientos y otros servicios) deben formar parte de este desarrollo que supone las TIC para adecuar el producto final a las demandas turísticas de la mejor manera posible.

Además, el uso de estas tecnologías potencia la gestión estratégica de estas organizaciones turísticas en el largo plazo por medio de la información que son capaces de suministrar por medio de colaboraciones y transacciones entre proveedores.

Las organizaciones turísticas han de recoger todos los elementos de su entorno, su competencia y las necesidades del cliente y reinventarse a sí mismos con el fin de mejorar su competitividad.

\section{Relación de las pymes del sector turístico con los clientes}

El mercado turístico está formado por muchos tipos de empresas, con diferentes tamaños, poder de mercado y enfoques distintos. Gracias a Internet se han podido eliminar las barreras de entradas que muchas empresas pequeñas tenían para poder acceder a los mercados. Anteriormente solo las grandes empresas eran capaces de llegar a un número grande de clientes, mientras que las pequeñas no podían permitirse ser visibles a tantos clientes potenciales. 


\begin{tabular}{|c|c|c|c|}
\hline \multicolumn{4}{|c|}{$\begin{array}{l}\text { TABLA } 1 \\
\text { TIPOS DE BÚSQUEDAS RELACIONADAS CON LA PLANIFICACIÓN DEL VIAJE EN EEUU } 2014 \\
\text { (En porcentaje) }\end{array}$} \\
\hline & $\begin{array}{c}\text { Al iniciar la planificación } \\
\text { del viaje }\end{array}$ & $\begin{array}{c}\text { Al considerar } \\
\text { varias posibilidades }\end{array}$ & Al realizar la reserva \\
\hline Términos relacionados con el destino ............... & 51 & 48 & 20 \\
\hline Términos relacionados con el precio ................. & 41 & 49 & 23 \\
\hline Nombres específicos de marcas o sitios web. & 31 & 48 & 30 \\
\hline Términos relacionados con actividades............ & 36 & 49 & 20 \\
\hline Términos relacionado con necesidades ........... & 32 & 43 & 22 \\
\hline
\end{tabular}

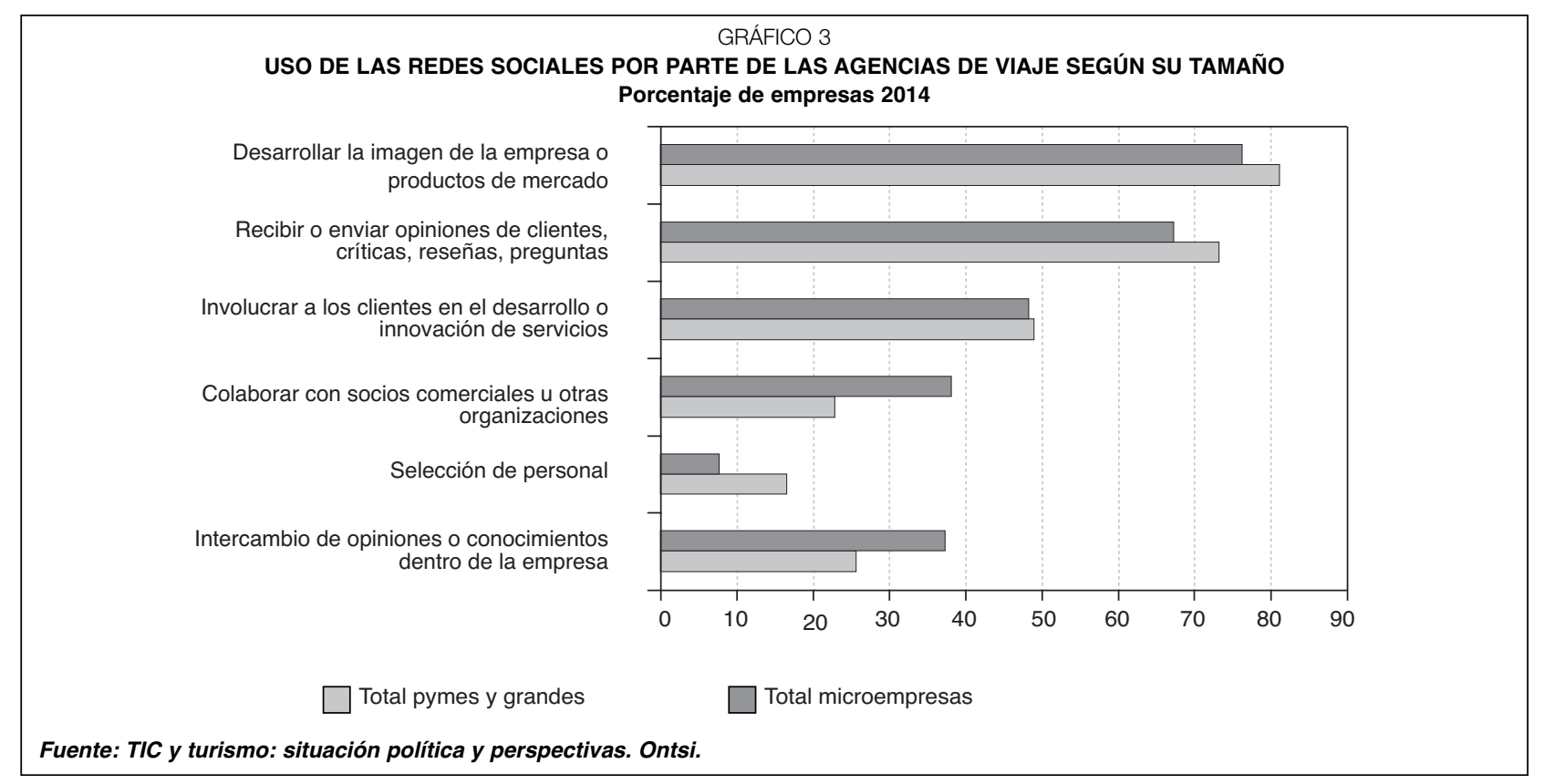

En la actualidad las pymes requieren menos presencia de personal y de recursos financieros para poder trabajar y competir en este sector turístico. Pero han de ser muy competitivas en otras cuestiones, por ejemplo, la información suministrada a los clientes, teniendo especial relevancia la correcta presentación y contar con contenidos actualizados. Deben tener especial cuidado en los siguientes aspectos:

- Una gestión sencilla y ágil del medio de contratación, medios de pago y ofrecer una seguridad en todos ellos. Cumpliendo con los estándares de seguridad de pago en Internet.

- Información correcta y detallada de los servicios ofertados. Intentando ser lo más completa posible (hoteles, viaje, sitios que visitar, clima...).
- Un procesamiento de la compra y confirmación de todos los servicios contratados lo más ágil posible.

- Un software de gestión de clientes (CRM) para tener una ventaja competitiva con respecto a la competencia.

- Análisis continúo de la información relativa a la pyme en Internet, donde se desarrolla la visibilidad de la empresa. Intentado conocer y gestionar todo lo que se dice de ella.

Todo esto nos permite descubrir un nuevo modelo de negocio de las pymes en el sector turístico, han de cambiar completamente la orientación actual de muchas de ellas para poder seguir compitiendo en el mercado actual. Es mucho más sencillo conocer con este modelo lo que los clientes o proveedores piensan $D$ 
de la empresa. También, es un tipo de empresa mucho más dinámico e innovador, con toda la información que posee puede modificar y ajustar su oferta y contenido a las tendencias que demanden sus clientes y proveedores. Se trata también de un proceso de modificación, aprendizaje y evolución continuo.

En el Gráfico 3 podemos comprobar cómo el uso de las redes sociales permite obtener información muy relevante a las empresas para modificar sus productos y además constatar el alto grado de uso que se hace de estas herramientas por parte de todas las empresas. No siendo especialmente relevante la diferencia entre grandes, pymes y microempresas.

\section{Relación de las pymes del sector turístico con los proveedores}

El uso de las nuevas tecnologías ha permitido la creación de redes de pymes dedicadas a las TIC, lo que da la posibilidad a estas pequeñas empresas de competir con las grandes empresas del sector turístico. Además, permite la unión de proveedores, socios y clientes en el diseño y creación de estas nuevas herramientas.

En un mercado tan competitivo como el del sector turístico los precios de los servicios se convierten en una de las principales herramientas para triunfar en este mercado. Aunque todo esto no ha terminado con el modelo tradicional de las agencias de viajes, operadores, oficinas de turismo y los mismos medios de contratación que se usaban antes de la llegada de Internet. Siguen siendo parte importante, aunque cada vez tienden a ser más especializadas y cuentan con una reputación que las precede. Lo mismo ocurre con los proveedores tradicionales Hertz, Hilton... aunque todos ellos han desarrollado un modelo en línea para poder ofrecer un mejor servicio a sus clientes.
Las nuevas formas de organización empresarial están haciendo cambiar la manera de competir de las empresas del sector turístico en el sector del comercio electrónico que se encuentra en un proceso de evolución constante.

Estas nuevas líneas de negocio, nuevos modelos basados principalmente en el uso de Internet como medio de comunicación, han entrado de lleno en todo el sector turístico modificándole la manera de interactuar con el cliente, proveedores y dentro de su propia empresa.

El futuro de este sector se presenta muy prometedor, cada vez más personas en el mundo van a realizar viajes, debido a los incrementos de renta de la población mundial y al incremento de demanda de actividades de ocio. Por lo que parece una buena idea posicionarse según la tendencia del sector turístico cada vez más competitivo, con mayor presencia de Internet y con grandes posibilidades de crecimiento para las pymes.

\section{Conclusión}

La industria del turismo está cambiando rápidamente, la manera de comunicarse los clientes y los proveedores ha evolucionado. El nuevo nexo de unión son las tecnologías de la información. Y el futuro pasa por comprender la evolución que están sufriendo estás tecnologías. Las empresas que sepan adaptarse a esta nueva manera de trabajar serán las que ganen en el mercado, las que den la espalda a estas tecnologías tendrán un futuro en el que, cada vez, tendrán menor importancia.

En este escenario las pymes pueden tener un futuro muy prometedor, por una parte, pueden aprovecharse de la especialización en determinados productos o bien intentar obtener beneficio anticipándose a las evoluciones que va a sufrir el mercado. En las nuevas estructuras $D$ 
empresariales van a predominar las pymes con pocos trabajadores, poco capital financiero y una gran capacidad de adaptación a las nuevas demandas de los clientes y de los proveedores. Todo ello debe estar sustentado en enseñar a los empresarios y trabajadores el nuevo modo de entender este mercado y la nueva manera de identificar las necesidades de los clientes y proveedores junto con los nuevos canales que emplean para comunicarse. Otra de las ventajas competitivas que deberán tener estas empresas será la capacidad de interpretación de estas nuevas demandas en las redes sociales, que se están transformando en los principales medios de obtención de información de gustos y necesidades de los clientes. Aquella empresa que sepa leer las demandas del cliente, sea capaz de ofertárselo y por último tenga la capacidad de ofrecérselo será la empresa ganadora del futuro en el sector turístico.

Por último, ha de plantearse como potencial mercado uno mucho más grande que el actual. Por medio de las TIC las ofertas pueden presentarse en cualquier parte del mundo y ser demandada por cualquiera, cerrarse la puerta de manera consciente a potenciales clientes no debe ser una opción ya que el coste, en la mayoría de los casos, no es elevado.

En otra línea se han de establecer estándares para el crecimiento de todas estas empresas que permitan dar una mayor seguridad a los clientes y eviten situaciones no deseadas. Las TIC suponen una fuente de competitividad y la posibilidad potencial de crecimiento de muchas pequeñas empresas que actualmente se encuentran dentro del modelo tradicional de negocio. El uso de estas tecnologías y los nuevos productos que irán apareciendo conducirán a una normalización de estas tecnologías, lo que permitirá un uso más seguro y una mayor predisposición de los clientes y proveedores a su uso.

\section{Bibliografía}

[1] CAMISÓN, C. Y MUÑOZ, C. (2015). La dirección económica y estratégica en los mercados turísticos. Madrid: EDIASA (Colección de Dirección de Empresas y Actividades Turísticas).

[2] EXCELTUR (2016). «Valoración Turística Empresarial de 2015 y Perspectivas para 2016». Informe Perspectivas Turísticas, $\mathrm{n}^{\circ} 55$.

[3] IPSOS MEDIACT (2014). «The 2014 Traveler's Road to Decision». Google Travel Study. Disponible en: https://storage.googleapis.com/think/docs/2014 -travelers-road-to-decision_research_ studies.pdf

[4] OBSERVATORIO NACIONAL DE LAS TELECOMUNICACIONES Y DE LA SI (2016). Tic $y$ turismo: situación política y perspectivas.

[5] OCDE. (2014). Tourism Trends and Policies 2014. Disponible en:

http://www.oecd.org/cfe/tourism/oecdtourism trendsandpolicies2014.htm

[6] ORGANIZACIÓN MUNDIAL DE TURISMO (2015). Recomendaciones de la OMT sobre accesibilidad de la información turística.

[7] RUIZ, L. (2010): «La Unión Europea en la Sociedad de la innovación y el Conocimiento. Plan Europe e iniciativa i2010». Revista Universitaria Europea, $n^{\circ} 12$, junio, pp. 87-98. 